\title{
Soliton Distribution in the Ball and Box Cellular Automation Model
}

\author{
Alpha Soko ${ }^{1,}$, , James Makungu ${ }^{2}$ \\ ${ }^{1}$ Department of Mathematics, Mzuzu University, Mzuzu, Malawi \\ ${ }^{2}$ Department of Mathematics, University of Dar es Salaam, Dar es Salaam, Tanzania
}

Email address:

alpha@aims.ac.tz (A. Sok)

${ }^{*}$ Corresponding author

\section{To cite this article:}

Alpha Soko, James Makungu. Soliton Distribution in the Ball and Box Cellular Automation Model American Journal of Mathematical and Computer Modelling. Vol. 4, No. 1, 2019, pp. 27-30. doi: 10.11648/j.ajmcm.20190401.14

Received: April 3, 2019; Accepted: May 16, 2019; Published: June 13, 2019

\begin{abstract}
Solitons are very important waves in Mathematical Physics. They help model many physical situations for instance tsunami waves, application in optical communication systems, application in plasma physics and application in laser guided technology among others. Despite enormous applications, solving the non-linear equations which have soliton solutions is challenging be- cause the nonlinear characteristic of the system abruptly changes due to some slight changes of valid parameters including time. A remedy to this is to use cellular automation models to model soliton dynamics. Cellular automation models do not change in response to slight changes on parameters. In this paper, using computer simulation, an investigation of the distribution of the number of solitons from the Ball and Box cellular automation model was examined. The distribution of the number of solitons from the two and three colour Ball and Box cellular automation was established. Using the online integer sequence, for the two colour Ball and Box cellular automation model, it was found that the distribution of the number of the solitons is indexed by the binomial coefficients. On the Other hand, for the three colour Ball and Box cellular automation model, it was found that only solitons of lengths one to four possesses the distribution while the other soliton lengths do not possess any distribution function.
\end{abstract}

Keywords: Solitons, Ball and Box Model, Cellular Automation, Integer Sequence, Binomial Coefficients

\section{Introduction}

The Ball and Box Soliton (BBS) is cellular automaton model which models soliton movement proposed by Takahashi and Satsuma $(1990)[1,2,3]$. Its state is defined by using an infinite array of boxes and a finite number of balls, with some boxes filled by balls bounded on the left side, but unbounded on the right side. Each box contains at most one ball. The time evolution of the value of the jth cell at time $t$, and state

$$
u_{j}^{t} \in(0,1)
$$

where it is assumed that $u_{j}^{t}=0$ for $\mathrm{j}$ far enough to the left and to the right is given by;

$$
u_{j}^{t+1}=\left\{\begin{array}{c}
1 \text { if } u_{j}^{t}=0 \text { and } \sum_{i=-\infty}^{j-1} u_{i}^{t}>\sum_{i=-\infty}^{j-1} u_{i}^{t+1} \\
0 \text { Otherwise }
\end{array}\right.
$$

This corresponds to taking a line of boxes, either containing a ball or empty and updating it. In other words, the rule corresponds to taking the leftmost ball out of its box and putting it in the first empty box to its right, then taking the new leftmost ball as long as it has not already been moved at this time step and moving it to the first empty box to its right. We continue with this process until each ball has been moved, this represents one-time step. The following example details the time evolution under the BBS; 


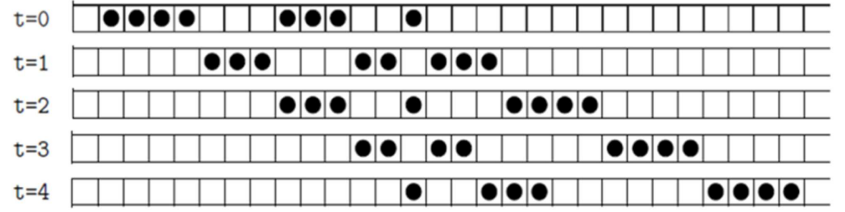

Figure 1. Time evolution in box and ball system.

We observe that consecutive balls of length four, three and one proceed to the right with constant speed unless they are too close to each other. The ball of length four is faster than the remaining two balls. Also, the ball of length two is faster than the ball of length one. We also observe that after some time, $0<t<4$, the balls collide. After the collision, there is phase shift; that is the balls have changed the orientation from four, three, one to one, three and four respectively. We note that the trajectory of the larger series has been shifted by four places to the right and the smaller one have been shifted by four places to the left.

Definition 1. 1 In Takahashi-Satsuma ball and box model, a soliton is consecutive length of balls that maintains its length after infinite time evolution [4].

From this definition, we see that in Figure 1 we have three solitons, that is soliton of length four, three and one respectively. We define speed of consecutive balls by the average number of boxes which the group passes per unit time and a basic ball as a ball $x$ which cannot be separated into balls $x_{1}$ and $x_{2}$ after a series of time evolution.

We denote a ball in box by 1 and empty box by 0 with this, another example of time evolution for the states is shown in Figure 2;

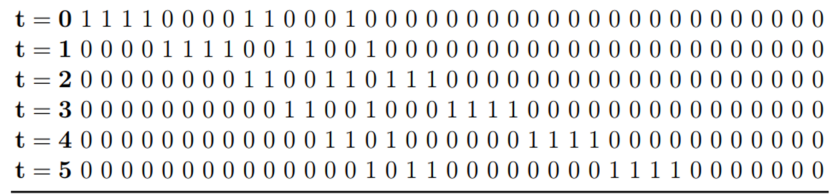

Figure 2. Time evolution in of three soliton states.

We see the series of lengths four, two and one from the left to right. After time evolution of $0<t<5$, there is phase shift of the series into the sequence of lengths one, two and four.

From the two examples above, we observe that when no interaction between balls, the balls move at speed equal to their length. During the time of evolution, balls collides with each other, and retain their original shapes. After the collision the balls change their original position. We also observe that if a ball of length $x$ passes through another ball of length $y$ where $x>y$, the phase shift of the longer ball is $+2 y$ and that of the shorter ball is $2 y$. These properties of the box-ball system are characteristic of the soliton systems. We summarize these characteristics into Theorem 1. 1 below:

Theorem 1. 1 [5, Theorem 2. 1] The basic solitons of boxball system are strings of consecutive balls; the soliton of length $\mathrm{k}$ has speed $\mathrm{k}$. When a system consisting of a disjoint union of basic solitons is allowed to scatter, the outcome is a sequence of basic solitons with the same set of lengths, arranged in non-decreasing order from left to right. Starting at any initial state, after a finite amount of time the system separates into basic solitons with non-decreasing length. Finally, if a soliton of length $\mathrm{x}$ passes through another soliton of length $y$ where $x>y$, the phase shift of the longer soliton is $+2 y$ and that of the shorter soliton is $-2 y$.

The BBS has been extensively investigated in the context of solitons and there is large literature on the subject. For instance Iwao (2009) [6, 7] studied the box-ball system with finitely many kinds of balls. The box-ball system was obtained from the hungry discrete Toda equation by ultradiscretization. They investigated the applications of the algebraic geometry and the tropical geometry to the ultradiscrete integrable system.

Also of significance notice on BBS is Mada et al. (2005) [8] who investigated conserved quantities of periodic boxball systems with arbitrary kinds of balls and box capacity greater than or equal to one. Finally, among the others, Fukuda et al. (2000) [9] studied the BBS in the crystal theory formulation. New conserved quantities and the phase shift of the solitons scattering were obtained by considering the energy function in the combinatorial $\mathrm{R}$ matrix.

Despite all this enormous investigation carried out, none of the researchers investigated the distribution of number of solitons from the multi-colour ball and box model. In this paper, we study the distribution of the number of solitons in the multi-color BBS model.

\section{Two Colour Ball and Box}

In this section the extended two color BBS model is investigated. We suppose that the box can hold at most one ball and there are two kinds of balls which are indexed by integers one and two. Then the time evolution rule corresponds to taking the leftmost ball of index one out of its box and putting it in the first empty box to its right, then taking the new leftmost ball of index one as long as it has not already been moved at this time step and moving it to the first empty box to its right. We continue with this process until each ball of index one has been moved, then we repeat this process to balls of index two. This represents one-time step. For example, consider the evolution of states in Figure 3 below for four-time steps.

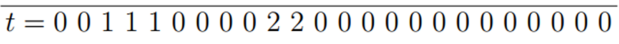

$$
\begin{aligned}
& t=10000111000022000000000000 \\
& t=2000000011100220000000000 \\
& t=3000000000000110011220000000 \\
& t=4000000000000011000122000 \\
& t=500000000000000110000122
\end{aligned}
$$

Figure 3. Time evolution in two color box and ball system.

In the example above, we had started with two configurations of lengths 3 and 2 . After time evolution, $0<t$ $<5$, there is phase shift of the lengths into the lengths 2 and 3 respectively. We also observe a mix up of the type of the balls. The mix up 122 moves with speed 3 so it is considered as one ball of length three. Using Theorem 1.1 it is easy to see that the two color BBS describes soliton dynamics. 
We now provide another example of the two-color state. We start with the arbitrary state 01222000000211.

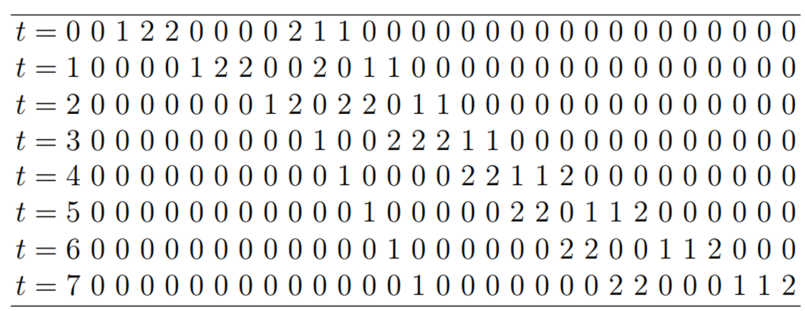

Figure 4. Time evolution in mixed state two color box and ball system.

From this example, it was observed that two unstable balls of lengths three unattained stability in the interval $0<t<7$. Using two unstable balls, we had formed three stable balls of lengths 1,2 and 3 respectively. Using Theorem 1. 1, we conclude that the three stable balls formed are solitons.

From the results of examples above, it is easy to see that different initial conditions of the soliton configurations produces different number of solitons. This calls for investigation of the distribution of the number of the solitons given a particular soliton configuration. we examine the distribution of number of solitons when we involve all possible initial soliton configuration in the two color BBS.

\subsection{Simulation of Two-Color Ball and Box}

On the 2 color BBS we simulated all the possible soliton configuration from the two color BBS model on the given $n$ empty boxes. We started with two empty boxes then produce all possible ways of coloring the boxes with two colors. Each of these possible ways represent one initial configuration. Using the produced initial configurations, we carry out time update on each of the configurations until stable length of balls are obtained. We then count the number of stable balls. We repeat the process for boxes more than two.

Here, a sequence of length $n$ shall represent coloring $n$ boxes with $n$ colors where the colors are the elements of the sequence, for instance the sequence 1212 shall mean color box one with color one, box two with color two, box three with color one and box four with color two.

As an example, for the two boxes with two colors, we have four ways of producing initial soliton configuration, 11, 22, 21,12 . In the configuration each state represents a unique soliton arrangement which we can start with.

\subsection{Results and Discussion}

Trivially for the one box we have two ways to color it with two colors either 1 or 2 . Both of these lead to 1 soliton. This means that the total number of 1 soliton is 2 . Continuing the process to the other number of boxes and using the on-line integer sequence [10], we have the following result;

Conjecture 2.1 Let $\mathrm{n}$ be the number of boxes and $\mathrm{k}$ be the number of solitons produced in the two color BBS. The two are related by the binomial coefficients;

$$
a(n, k)=\left\{\begin{array}{c}
\left(\begin{array}{c}
n+1 \\
2 k-1
\end{array}\right) \text { if } n+1 \geq 2 k-1 \\
0 \text { else }
\end{array}\right.
$$

provided that $\mathrm{n}, \mathrm{k} \in \mathrm{Z}+$.

\section{Three Colour Ball and Box System}

We now extend the model introduced in previous section by introducing more species of the ball. We suppose that the box can hold at most one ball and there are three kinds of balls which are indexed by integers one to three.

The time evolution rule corresponds to taking the leftmost ball of index one out of its box and putting it in the first empty box to its right, then taking the new leftmost ball of index one as long as it has not already been moved at this time step and moving it to the first empty box to its right. We continue with this process until each ball of index one has been moved, then we repeat this process to balls of index two and index three. This represents one-time step.

\subsection{Simulation of Three Colour Ball and Box Model}

As in previous section, we start with two empty boxes then produce all possible ways of coloring the two boxes with three colors. This represent initial soliton configuration. We then evolve all initial soliton configurations to count how many solitons of each kind results. We repeat this process for boxes more than two.

Let 1 denote colour number one, 2, colour number two and 3 represents colour number three. Then 12 denotes box 1 colored with colour one and box two colored with colour two. For the two boxes we have 9 ways of producing initial soliton configuration. We continue with this until all states are filled up. The results of this section are given in Section 3.2 below.

\subsection{Results and Discussion}

Using the on-line integer sequence and data for the three colour BBS, the Conjecture 3.1 below gives the distribution of the number of solitons.

Conjecture 3.1 Let $n$ be the number of boxes and $k$ be the number of solitons produced in the three color BBS. The two are related by the binomial coefficients.

$$
a(n, k)=\left\{\begin{array}{c}
\left(\begin{array}{c}
n+2 \\
2
\end{array}\right) \text { if } k=1 \\
3\left(\begin{array}{c}
n+3 \\
5
\end{array}\right)-2\left(\begin{array}{c}
n+2 \\
5
\end{array}\right) \text { if } k=2 \\
\left(\begin{array}{c}
n+1 \\
4
\end{array}\right)+10\left(\begin{array}{c}
n+1 \\
5
\end{array}\right)+15\left(\begin{array}{c}
n+1 \\
5
\end{array}\right)+7\left(\begin{array}{c}
n+1 \\
7
\end{array}\right)+\left(\begin{array}{c}
n+1 \\
8
\end{array}\right) \text { if } k=3
\end{array}\right.
$$

No pattern elsewhere detected which implies there is no

specific sequence which can fit the data. 


\section{Conclusion}

In this paper we have successfully investigated the soliton distribution in the two- and three-color Ball and Box model. The soliton distribution on the two-color Ball and Box automation model is index by binomial coefficients while for the three-color model there is distribution only for at most three solitons while for more than three solitons there is no distribution function. An advantage to use the Ball and Box automation model is that we prevent dealing with non-linear systems which challenging to model solitons.

Since the BBS is composed of a finite number of boxes and balls, it can only take on a finite number of patterns. Hence its trajectory is always simple. In the case where the box capacity is one everywhere and only one kind of ball exists, the formula used to calculate the fundamental cycle is explicitly obtained $[10,11]$ Hence, using the results in this paper, we may get the formula to calculate number of solitons for multi-color solitons for two colors. In the future we wish to address problem of finding distribution functions for multi-color solitons with colors more than 3

\section{Acknowledgements}

The authors are grateful to Professor Patrick Dorey for helpful discussions on the present work. This work was supported in part by African Institute of Mathematical Sciences (AIMS).

\section{References}

[1] D. Takahashi, J. Satsuma, A soliton cellular automaton, Journal of the Physical Society of Japan 59 (10) (1990) 35143519.

[2] T. Tokihiro, D. Takahashi, J. Matsukidaira, J. Satsuma, From soliton equations to integrable cellular automata through a limiting procedure, Physical Review Letters 76 (18) (1996) 3247.

[3] M. Torii, D. Takahashi, J. Satsuma, Combinatorial representation of invariants of a soliton cellular automaton, Physica D: Nonlinear Phe- nomena 92 (3-4) (1996) 209-220.

[4] P. Caudrey, Memories of hirota's method: application to the reduced Maxwell-bloch system in the early 1970s, Philosophical Transactions of the Royal Society of London A: Mathematical, Physical and Engineering Sciences 369 (1939) (2011) 1215-1227.

[5] T. Lam, P. Pylyavskyy, R. Sakamoto, Box-basket-ball systems., Reviews in Mathematical Physics 24 (08) (2012) 1250019 .

[6] S. Iwao, The periodic box-ball system and tropical curves., RIMS K^okyu^roku Bessatsu B 13 (2009) 157-174.

[7] A. Kuniba, T. Takagi, Bethe ansatz, inverse scattering transform and tropical riemann theta function in a periodic soliton cellular automa- ton for $\mathrm{a}^{\wedge}(1) \mathrm{n}$, Symmetry, Integrability and Geometry: Methods and Applications $6(0)$ (2010) 13-52.

[8] J. Mada, M. Idzumi, T. Tokihiro, Path description of conserved quanti- ties of generalized periodic box-ball systems., Journal of mathematical physics 46 (2) (2005) 022701 .

[9] K. Fukuda, Y. Yamada, M. Okado, Energy functions in box ball sys- tems., International Journal of Modern Physics A 15 (09) (2000) 1379- 1392.

[10] N. J. Sloane, et al., The on-line encyclopedia of integer sequences (2003). URL https://oeis.org/.

[11] A. Kuniba, M. Okado, R. Sakamoto, T. Takagi, Y. Yamada, Crystal interpretation of kerov-kirillov-reshetikhin bijection., Nuclear Physics B 740 (3) (2006) 299-327.

[12] A. Kuniba, R. Sakamoto, Combinatorial bethe ansatz and generalized periodic box-ball system., Reviews in Mathematical Physics 20 (05) (2008) 493-527. 